\title{
Symptoms of psychological distress among bereaved relatives of deceased patients in the intensive care unit during the first COVID-19 wave
}

\author{
Margo M. C. van Mol ${ }^{1 *} \mathbb{0}$, Sebastian Wagener ${ }^{1}$, Judith Rietjens ${ }^{2}$ and Corstiaan A. den Uil ${ }^{3,4}$
}

(๑) 2022 Springer-Verlag GmbH Germany, part of Springer Nature

\section{Dear Editor,}

During the coronavirus disease 2019 (COVID-19) pandemic, policies of family-centered care in the intensive care unit (ICU) were impacted by visiting restrictions and undesirable physical distance to the patient [1]. This might have increased psychological distress, including complicated grief (CG) among bereaved relatives [2]. CG is characterized by intense symptoms of grief, separation distress, intrusive thoughts, and feelings of emptiness or meaninglessness lasting for more than 6 months post-loss [3]. A French qualitative study reported that relatives' experiences of ICU care and mourning were disrupted during the pandemic [4]. We aimed to compare symptoms of CG and psychological distress such as depression, anxiety and posttraumatic stress in bereaved relatives of deceased ICU patients before and during the COVID-19 pandemic. To the best of our knowledge, this is the first study exploring a hypothesized raise in symptoms of CG in bereaved relatives as a result of the COVID-19 pandemic.

This cross-sectional survey study is part of the BRICstudy [5]. We stratified the post-intervention group by date of patient's deaths: September 2019-February 2020 (non-COVID-group; $n=114$, patients dying before the pandemic) and March-May 2020 (COVID-group; $n=43$, patients dying during the 1st wave). All first and second contact persons were approached for study participation

\footnotetext{
*Correspondence: m.vanmol@erasmusmc.nl

${ }^{1}$ Department of Intensive Care Adults, Erasmus MC, University Medical Center, Rotterdam, The Netherlands
}

Full author information is available at the end of the article $(n=269)$. After providing informed consent, relatives were invited to complete a questionnaire measuring CG, anxiety, depression, and posttraumatic stress 8 weeks after bereavement.

In total, $121(45 \%)$ respondents completed the questionnaire (non-COVID-group $n=86$, COVID-group $n=35)$. Most respondents were partners $(52 \%)$, female (71\%), and had a mean age of 52 years (range 20-83). We found no difference between groups regarding age, relationship to the patient and religion. Symptoms of CG were above the cutoff in $1.3 \%$ in the non-COVID-group and $48.6 \%$ in the COVID-group. All outcome variables showed a normal distribution, with both skewness and kurtosis between 1 and -1 . Therefore, using a Student's $T$-test was allowed. Table 1 shows levels of psychological distress indicating significant worse results for the COVID-group compared to the non-COVID-group.

Our study has limitations. First, we measured CG earlier than 6 months post-death, potentially leading to overestimation. However, as the measurements were conducted at similar moments in the non-COVID-group and COVID-group, the comparison is meaningful. Second, during the pandemic, visiting hours in our ICU were reduced from almost all day (11.00 AM-21.00 PM) to maximum $1 \mathrm{~h}$ in person for two relatives per patient. Although all relatives were physically present during the dying process, visiting restrictions could have impacted relatives' psychological distress. Watchful waiting and coping mechanisms are common strategies to recover. However, if this period was overlapping with start of the pandemic, which was the case for $27 \%$ of bereaved relatives in the non-COVID-group, the process could be disrupted by traumatic circumstances and less social 
Table 1 Levels of psychological distress in the non-COVID-group and the COVID-group

\begin{tabular}{|c|c|c|c|c|c|c|}
\hline & \multicolumn{2}{|l|}{ Mean $( \pm S D)$} & \multirow[t]{2}{*}{ Standardizer ${ }^{\mathrm{a}}$} & \multirow[t]{2}{*}{ Point estimate } & \multicolumn{2}{|c|}{$95 \% \mathrm{Cl}^{\mathrm{b}}$} \\
\hline & Non-COVID & COVID & & & Lower & Upper \\
\hline$C G^{*}$ & $37.4 \pm 8.8$ & $60.9 \pm 9.4$ & 8.95 & -2.61 & -3.14 & -2.09 \\
\hline Anxiety** & $6.9 \pm 2.3$ & $9.2 \pm 1.9$ & 2.16 & -1.08 & -1.55 & -0.6 \\
\hline Depression** & $9.4 \pm 2.4$ & $11.8 \pm 1.6$ & 2.16 & -1.12 & -1.61 & -0.62 \\
\hline $\mathrm{PTS}^{* * *}$ & $31.6 \pm 10.5$ & $56.2 \pm 11.2$ & 10.68 & -2.3 & -2.83 & -1.78 \\
\hline
\end{tabular}

$S D$ standard deviation to the mean, $\mathrm{Cl}$ confidence interval

a Standardizer as the denominator used in estimating the effect sizes

b Independent samples effect sizes with Cohen's $d$ using a Student's $T$-test analysis

${ }^{*} \mathrm{CG}=$ symptoms of complicated grief measured after 8 weeks with traumatic grief inventory-self report version, 19 items, scores range from 17 minimum to 85 maximum

**Anxiety and depression symptoms measured with Hospital Anxiety and Depression Scale, 7 items in each subscale, scores range from 0 minimum to 21 maximum

***PTS $=$ symptoms of posttraumatic stress measured with Impact of Events Scale, 22 items, scores range from 0 minimum to 88 maximum

support. Unmeasured confounders, such as lack of social support, confrontations with COVID-19 due to ongoing restrictions, and media reports, could have contributed to psychological distress in both groups differently.

Our findings emphasize the need for unrestricted visiting policies and social support for bereaved relatives, who are at high risk of developing psychological distress.

\section{Trial registration}

Netherlands Trial Register NL 7875, Registered on 27/07/2019, http://www.trialregister.nl.

\section{Author details \\ ${ }^{1}$ Department of Intensive Care Adults, Erasmus MC, University Medical Center, Rotterdam, The Netherlands. ${ }^{2}$ Department of Public Health, Erasmus MC, University Medical Center, Rotterdam, The Netherlands. ${ }^{3}$ Department of Cardiology and Intensive Care, Erasmus MC, University Medical Center, Rot- terdam, The Netherlands. ${ }^{4}$ Department of Intensive Care, Maasstad Hospital, Rotterdam, The Netherlands.}

\section{Acknowledgements}

The authors would like to thank all participating bereaved relatives and the Department of Intensive Care Unit Adults of Erasmus MC, the Netherlands for their involvement in the study.

\section{Author contributions}

MMCvM, CAdU and JR jointly designed the study, raised funding and established the development of the study protocol. MMCVM and SW prepared the study materials and produced the first draft of the article outline. All the authors (MMCVM, SW, CAdU, and JR) contributed substantially to the analyses of literature, critically revised the content of the manuscript, have read and approved the final version.

\section{Funding}

This work has been supported by a grant from Erasmus MC "Evidence Based Care by Nurses", project 2017-17407, which had no role in the design of this study and has no role in its execution, analysis and interpretation of data, or publication of results.

\section{Data availability}

Data are available upon reasonable request.

\section{Conflicts of interest}

There are no conflicts of interest to declare.

\section{Ethical approval}

The study was conducted in accordance with the amended Declaration of Helsinki and in accordance with the Dutch Medical Research Involving Human Subjects Act. The Medical Ethical Review Committee of Erasmus Medical Centre Rotterdam approved the study (MEC-2018-1598). The study complied with the Netherlands Code of Conduct for Scientific Practice from the Association of Universities in the Netherlands (VSNU). Participants were informed about the study both orally and by letter.

\section{Consent to participate}

A written informed consent was obtained from all the participants.

\section{Publisher's Note}

Springer Nature remains neutral with regard to jurisdictional claims in published maps and institutional affiliations.

Accepted: 13 January 2022

Published: 28 January 2022

\section{References}

1. Jeitziner MM, Camenisch SA, Jenni-Moser B, Schefold JC, Zante B (2021) End-of-life care during the COVID-19 pandemic-What makes the difference? Nurs Crit Care 26(3):212-214. https://doi.org/10.1111/nicc.12593

2. Diolaiuti F, Marazziti D, Beatino MF, Mucci F, Pozza A (2021) Impact and consequences of COVID-19 pandemic on complicated grief and persistent complex bereavement disorder. Psychiatry Res 300:113916. https:// doi.org/10.1016/j.psychres.2021.113916

3. Downar J, Koo E, des Ordons AR, Smith O, Cook D, Golan E et al (2018) Prevalence and predictors of severe grief reactions and desire for support following a death in the intensive care unit: a multicentre observational study. Intensive Care Med 44(4):521-522. https://doi.org/10.1007/ s00134-017-5027-2

4. Kentish-Barnes N, Cohen-Solal Z, Morin L, Souppart V, Pochard F, Azoulay E (2021) Lived experiences of family members of patients with severe COVID-19 who died in intensive care units in France. JAMA Netw Open 4(6):e2113355. https://doi.org/10.1001/jamanetworkopen.2021.13355

5. van Mol MM, Wagener S, Latour JM, Boelen PA, Spronk PE, Corstiaan A et al (2020) Developing and testing a nurse-led intervention to support bereavement in relatives in the intensive care (BRIC study): a protocol of a pre-post intervention study. BMC Palliat Care 19(1):1-10. https://doi.org/ 10.1186/s12904-020-00636-8 